NASZA DERMATOLOGIA Online OUR DERMATOLOGY Online

Source of Support Nil

Competing Interests: None

\section{ZOSTERIFORM LICHEN PLANUS: CASE REPORT OF A} RARE VARIANT OF LICHEN PLANUS

\author{
Kanthilatha Pai ${ }^{1}$, Sathish Pai ${ }^{2}$ \\ ${ }^{I}$ Department of Pathology, KMC International center, Manipal University, Manipal, \\ India \\ ${ }^{2}$ Department of Dermatology, KMC Manipal, Manipal University, Manipal, India
}

Corresponding author: Dr Kanthilatha Pai

klpai@yahoo.com

\begin{abstract}
Since its original description by Devergie in 1854, several cases of linear lichen planus have been described in the literature, but there have been notably few cases of the more rare zosteriform lichen planus. Zosteriform lichen planus needs to be differentiated from linear lichen planus and other linear dermatoses. We present a case of Zosteriform Lichen planus for its rarity and briefly review literature.
\end{abstract}

Key words: zosteriform; linear; lichen planus

\section{Introduction}

Lichen planus (LP) has certain clinical variants which may present difficulty in diagnosis especially when the lesions happen to be arranged in a linear fashion. Linear distribution of the disease has been described in less than $1 \%$ of patients and need to be differentiated from other linear dermatoses [1]. It may be zosteriform and follows the lines of Blaschko [2,3]. We report a rare case of Zosteriform variant of Lichen planus in a young patient.

\section{Case Report}

A 12-year-old boy presented with multiple pruritic, violaceous annular skin lesions over the left side of chest. It started as small violaceous papules which coalesced to form ring lesions. There was no past history of herpes zoster or any other skin lesions. Family history was not significant. Dermatological examination revealed multiple, violaceous annular lesions in T5 dermatomal region over the chest, extending from below the left nipple to the left lateral side of chest, measuring more than $2 \mathrm{cms}$ in diameter (Fig. 1). The margin of the lesion was beaded and the center atrophic. Few flat topped, violaceous, polygonal papules were present on the skin between the annular lesions. No other skin lesions were noted any where in the body.

The histopathologic examination of the lesion showed orthokeratotic hyperkeratosis, variable acanthosis, focal hypergranulosis, hydropic degeneration of basal cell layer and band like inflammatory infiltrate, suggestive of Lichen Planus (Fig. 2).

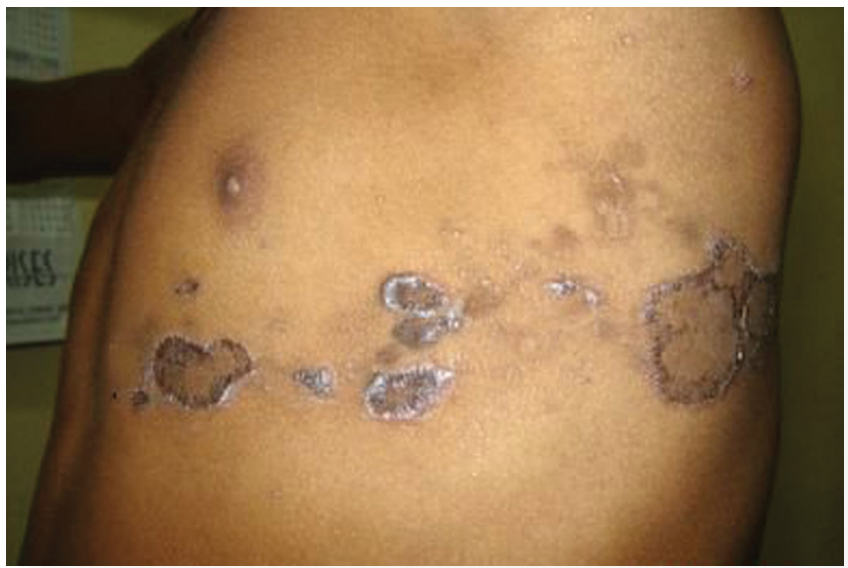

Figure 1. Multiple voilaceous papules and plaques over T5 dermatome

\section{Disuussion}

Lichen planus (LP) is an idiopathic inflammatory disease of the skin and mucous membrane. It is characterized by pruritic violaceous papules that favor the extremities.

In addition to the classical appearance, about 20 different variants are described [4]. Linear LP refers to lichen planus with a unilateral linear distribution and may occur at the site of healed zoster [5]. Although it is common in children but is also seen in adults [6]. Zosteriform pattern is a variant of LP that occurs without evidence of herpes zoster, and is extremely rare in occurrence. 


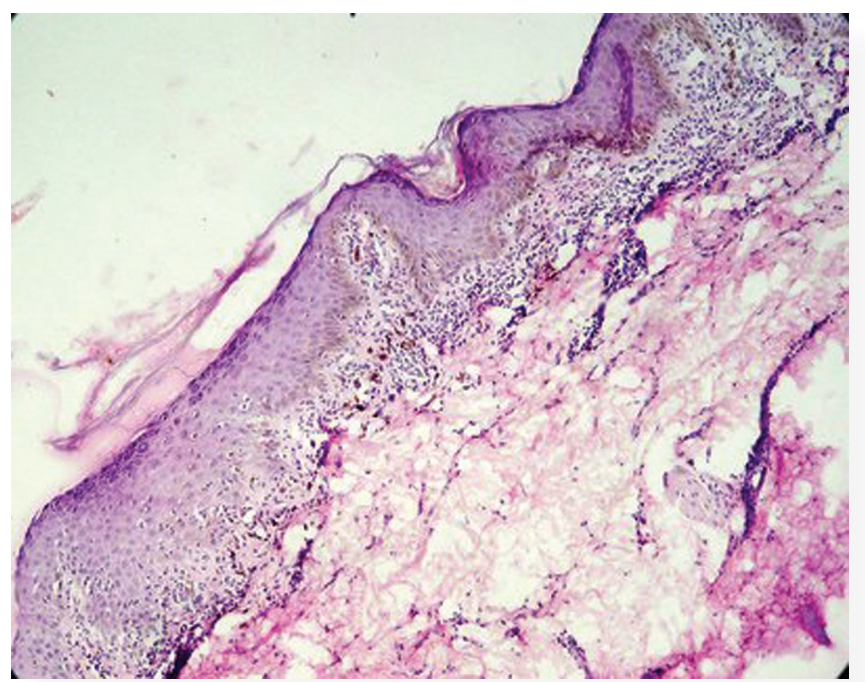

Figure 2. Photomicrograph showing hyperkeratosis, irregular acanthosis, basal cell degeneration and lichenoid dermal infiltrate, $\mathrm{H} \& \mathrm{E}, 100 \mathrm{X}$

There is a definite distinction between the linear and the zosteriform type of lichen planus, to which there has not been strict adherence. In the former condition, the papular lesions appear as narrow lines about 1 or $2 \mathrm{~cm}$. wide, which may follow the course of a nerve, of a vein or of a lymphatic vessel or one of Voigt's lines. In the latter the lesions form a band several centimeters wide that follows the course of a peripheral cutaneous nerve and its branches or appears over areas of radicular nerve distribution. Zosteriform LP also needs to be differentiated clinically from zona zoster and other linear dermatoses. It is an extremely pruritic variant of Lichen Planus.

The zosteriform arrangement of lichenoid papules is rare and is interpreted

as a cutaneous reaction possibly triggered by some neural factor [7].

Many disorders occur within an area that is innervated by a particular spinal cutaneous nerve. Such a distribution of lesions has provoked many authors to suggest a theory of neural origin to the linear/zosteriform lichen planus, on the other hand it has been recently suggested that most of the lesions occurring in so-called zosteriform manner do not follow a dermatomal pattern or apparently a nerve segment but are rather along the Blaschko's line [5]. Some authors believe that true zosteriform LP only exists in cases who have developed lesions on the sites of healed herpes zoster [8].

The histology of linear LP is characteristic and enables distinction from other linear

dermatoses such as lichen striatus, linear nevi and linear psoriasis.

Treatment modalities include topical moderate to high potency corticosteroids, topical salicylic acid, and systemic sedative antihistamines. In unresponsive cases systemic corticosteroids or intralesional corticosteroids can be instituted.

Paediatric aspect of the disease: Children should be allowed to engage in full activities, and to attend school. However, because lichen planus demonstrates the isomorphic response, attempts should be made to minimize trauma to the skin. Children and parents should be informed that lichen planus can be a chronic disorder, and that numerous recurrences may occur over the next several months to years.

\section{REFERENCES}

1. Brownstein MH, Silverstein L, Lefing W: Lichenoid epidermal nevus: "Linear lichen planus". J Am Acad Dermatol. 1989;20:9135.

2. Ang P, Tay YK, Giam YC: Pruritic linear eruption on a child. Arch Dermatol. 2001; 137:85-90.

3. Kabbash C, Laude TA, Weinberg JM, Silverberg NB: Lichen planus in the lines of Blaschko. Pediatr Dermatol. 2002;19:541-5.

4. Boyd AS, Neldner KH: Lichen planus. J Am Acad Dermatol. 1991;25:593-619.

5. Turel A, Ozturkcan S, Ozturkcan S, Sahin MT: Wolf's isotopic response: a case of zosteriform lichen planus. J Dermatol. 2002;29:339-42.

6. Long CC, Finlay AY: Multiple linear lichen planus in the lines of Blaschko. Br J Dermatol. 1996;135:275-6.

7. Happle R: Zosteriform' Lichen planus: Is it Zosteriform? Dermatology. 1996;192:385-6.

8. Braun RP, Barua D, Masouye I: Zosteriform lichen planus after herpes zoster. Dermatology. 1998;197:87-8.

Copyright by Kanthilatha Pai, et al. This is an open access article distributed under the terms of the Creative Commons Attribution License, which permits unrestricted use, distribution, and reproduction in any medium, provided the original author and source are credited. 